\title{
Magnetic Field Strengths of GPS Radio Sources
}

\author{
V. G. Panajyan
}

Byurakan Astrophysical Observatory, Armenia.

$\mathrm{GHz}$ peaked spectrum radio sources (GPS) are believed to be a subclass of compact steep spectrum radio sources (CSS) with high frequency spectral indices $\alpha<-0.5\left(S \sim \nu^{\alpha}\right)$, linear sizes of pc to kpc scale and turnover spectra near $1 \mathrm{GHz}$. Due to the work of many radioastronomers during the past two decades many properties of CSS and GPS radio sources at present are known (O'Dea,C.P. et al.1998, and references therein).

The origin of the low frequency turnover of the radio continuum spectra is disputable: there are mainly two ways to explain the low frequency turnover of the radio sources - 1 . free-free absorption of the radiation and 2. synchrotron self absorption. For the latter case an equation was derived by V. Slish (Slish 1963) and by I.P.Williams (Williams 1963), connecting angular sizes of the radio sources with turnover frequency $\nu_{t o}$, turnover flux density $S_{t o}$, redshift $z$ and magnetic field strength $B$. This equation was used by many authors to estimate magnetic field strengths B of radio sources (for example, Artyukh et al. 1994a, $1994 \mathrm{~b}$ ). Recently I have completed a sample of GPS radio sources of intermediate flux densities (Panajyan 1988) containing 30 GPS radio sources. Unfortunately the data on the linear sizes of all radio sources of this sample and redshifts of most of them are unknown, therefore it is not possible to estimate their magnetic field strengths. In this paper I estimate magnetic field strengths of radio sources of the complete sample of strong GPS radio sources of Stanghellini and coworkers (Stanghellini et al. 1988) assuming that the low frequency turnover of the spectra is due to synchrotron self absorption. I have used the modified version of the synchrotron self absorption equation used by M.J.A. Oort (Oort 1988). From the equations for angular sizes and linear sizes from that paper for magnetic field strength $B$ one can write

$$
B=\left((1+z)^{2} \nu_{t o}^{1.25} D / 0.85 z(1+z / 2) S_{t o}^{0.5}\right)^{4},
$$

(the case, when $H=50 \mathrm{~km} / \mathrm{sMpc}, q_{0}=0$ ), where $B$ is in $10^{-4} G, S_{t_{o}}$ is in $\mathrm{mJy}$, $\nu_{t_{0}}$ is in $\mathrm{GHz}$ and $D$ is in $p c$. The results of calculations are as follows. The values of magnetic field strengths of the complete sample of GPS radio sources spread in a broad region: from $10^{3}$ to $10^{-5} G$, the median value of this sample is equal to $6 G$, only for $40 \%$ of radio sources $B<1 G$. The values $B>1 G$ are too large to be real. Note that Athreya and coworkers got $1 G$ for the steep spectrum radio cores' magnetic field strengths (Athreya 1997).

It is known that the accuracy of estimation of magnetic field strengths using equation mentioned above is not high; the errors range up to two orders. The largest contribution in the error is due to the linear sizes of the radio sources: the uncertainty is proportional to the third power of the linear sizes. Therefore 


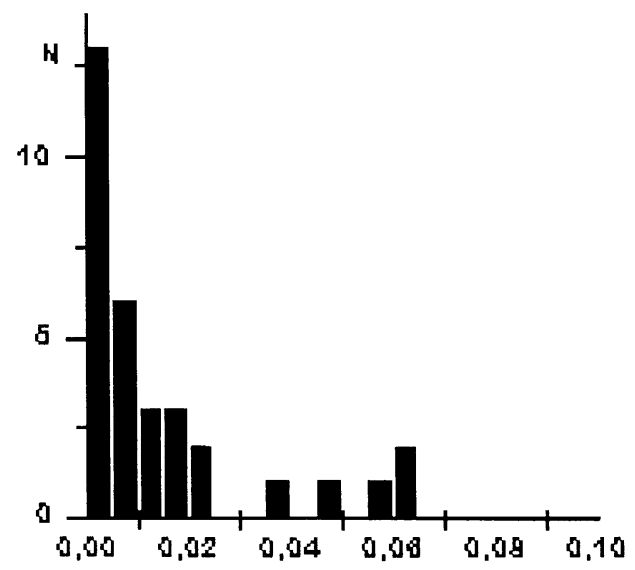

Figure 1. The histogram of the ratio $B^{0.25} / D$.

we calculated the ratio $B^{0.25} / D$ from the equation used above

$$
B^{0.25} / D=(1+z)^{2} \nu_{t o}^{1.25} / z(1+z / 2) S_{t o}^{0.5}
$$

This ratio shows $B^{0.25}$ for angular size of $1 p c$. But the magnetic field strength's connection with linear sizes is not simple, because linear sizes of the complete sample of GPS radio sources statistically are connected to the turnover frequency as follows (O'Dea C.P. 1997):

$$
\nu_{t o} \sim D^{-0.65}
$$

Fig. 1 shows the histogram of the $B^{0.25} / D$. One can see concentration of the values of the ratio $B^{0.25} / D$ around the median 0.0073 .

\section{References}

Arthyukh, V. S., Hovhannissian, M. A., \& Tyul'bashev, S. A. 1994, Pisma v AZh. 20, 178

Arthyukh, V.S., Hovhannissian, M. A., \& Tyul'bashev, S. A. 1994, Pisma v AZh. 20, 258

Athreya, R. M., Kapahi, V. K., McCarthy, P.J., \& van Breugel, W. 1997, MNRAS, 289, 525

O'Dea, C. P.,1998, PASP, 110, 493

O'Dea, C. P., \& Baum, C. A. 1997, AJ, 113, 148

Oort, M. J. A. 1988, A\&A, 192, 42

Panajyan, V. G. 1998, Astrofizika, 41, 377

Slish, V. 1963, Nature, 199, 682 
Stanghellini, C., O'Dea C. P., Dallacasa, D., Baum, S. A., Fanti, R., \& Fanti, C. 1998, A\&AS, 131,303

Williams, I. P. 1963, Nature, 200, 56 